\title{
Cascading Gravity and Degravitation
}

\author{
Claudia de Rham \\ Dept. of Physics \& Astronomy, McMaster University, Hamilton ON, Canada \\ Perimeter Institute for Theoretical Physics, Waterloo, ON, Canada
}

\begin{abstract}
Cascading gravity is an explicit realization of the idea of degravitation, where gravity behaves as a high-pass filter. This could explain why a large cosmological constant does not backreact as much as anticipated from standard General Relativity. The model relies on the presence of at least two infinite extra dimensions while our world is confined on a four-dimensional brane. Gravity is then four-dimensional at short distances and becomes weaker and weaker at larger distances.
\end{abstract}

Keywords: dark energy, massive gravity, large extra dimensions, six-dimensional gravity

PACS: $11.10 . \mathrm{Gh}, 11.10 . \mathrm{Kk}, 11.25 . \mathrm{-W}$

\section{Degravitation and massive gravity}

The cosmological constant problem is one of the most tantalizing puzzles of modern cosmology. While most approaches propose an explanation for what dark energy is, and why the Universe is accelerating, very little progress has been made in understanding why the observed cosmological constant is so much smaller than expected in the first place. Instead, we follow here an alternative approach based on the idea of degravitation, see ref. [1], where the cosmological constant could be as large as expected from standard field theory, but would simply gravitate very little. In models where the graviton has a mass, gravity is typically weaker in the infrared (IR) and could play the role of a highpass filter.

The idea behind degravitation is to promote the mass of the graviton in the Fierz-Pauli action to an operator $m^{2}(\square)=\left(L^{2} \square\right)^{\alpha} / L^{2}$, such that the inverse propagator is then of the form

$$
\mathscr{G}^{-1}=\square\left(1-\frac{m^{2}(\square)}{\square}\right)=\square\left(1-\left(L^{2} \square\right)^{\alpha-1}\right) .
$$

To satisfy unitarity, the power $\alpha$ should be positive, while to represent an IR modification of gravity one should have $\alpha<1$. One can further show that in order for the resulting theory to play the role of a high-pass filter, the power should satisfy $\alpha<1 / 2,[1,2]$. The extra-dimensional DGP scenario proposed by Dvali, Gabadadze and Porrati, see ref. [3], represents a specific model of massive gravity, with the parameter $\alpha=1 / 2$. Lying precisely on the border, this model does not represent an explicit non-linear realization of degravitation but is worth studying as it provides valuable intuition. We will then extend the DGP scenario to higher codimensions and present the first non-linear realization of a high-pass filter for a spin-2 particle. 


\section{DGP as an example of massive gravity}

The DGP scenario relies on the idea that our four-dimensional world could be a hypersurface or brane embedded in a five-dimensional space-time. If the volume of the extra dimension were finite, the effective four-dimensional theory would admit a separate zero-mode that would dominate in the IR. In order to represent a modification of gravity in the IR, it is therefore imperative to keep the volume of the extra dimension infinite. Without any further ingredient, gravity would then be five-dimensional at all scales, and clearly incompatible with Newton's law. To recover four-dimensional gravity at short scales, the DGP model relies on a four-dimensional Einstein-Hilbert term localized on the brane, leading to the action

$$
S_{\mathrm{DGP}}=\int \mathrm{d}^{5} x \sqrt{-g_{5}} \frac{M_{5}^{3}}{2} R^{(5)}+\int \mathrm{d}^{4} x \sqrt{-g_{4}}\left(\frac{M_{4}^{2}}{2} R^{(4)}+\mathscr{L}_{m}\right),
$$

where $M_{n}$ is the $n$-dimensional Planck mass, $R^{(n)}$ the scalar curvature with respect to the $n$-dimensional metric, and $\mathscr{L}_{m}$ the Lagrangian of matter fields localized on the brane. In five-dimensions, gravity is propagated by a spin-2 massless field which has five degrees of freedom. From a four-dimensional point of view, on the other hand, these five degrees of freedom will play the role of a massive spin-2 particle, and the effective theory on the brane will be an explicit non-linear realization of massive gravity. The effective fourdimensional propagator in this model is then

$$
\mathscr{G}(k)=\frac{1}{M_{4}^{2}} \frac{1}{k^{2}+m_{5} k}
$$

where $k$ is the four-dimensional momentum and the "graviton mass" is $m_{5}=M_{5}^{3} / M_{4}^{2}$. This propagator is precisely of the form presented in (1), with the parameter $\alpha=1 / 2$. At short distances compared to $1 / m_{5}$, the four-dimensional curvature terms dominate and gravity appears four-dimensional, with the standard $1 / r^{2}$ Newton's law. At larger distances, on the other hand, gravity probes the extra dimension and gravity behaves fully five-dimensional, with force law going as $1 / r^{3}$, gravity is therefore successfully modified in the IR. As mentioned in the previous section, this modification of gravity is however not sufficient To represent an explicit realization of a high-pass filter, and we now turn to a higher dimensional generalization of the DGP model.

\section{Higher codimension sources}

In a $(4+n)$-dimensional space-time, the gravitational potential goes as $V(r) \sim$ $r^{-(1+n)}$. In the spectral representation of the Newtonian propagator

$$
V(r)=\int_{0}^{\infty} \frac{\rho\left(m^{2}\right) e^{-m r}}{r} \mathrm{~d} m^{2}
$$


this corresponds to a spectral density $\rho\left(m^{2}\right) \sim m^{n-2}$ as $m \rightarrow 0$. The propagator then has a spectral representation

$$
\mathscr{G}(k)=\int_{0}^{\infty} \frac{\rho\left(m^{2}\right)}{k^{2}+m^{2}} \mathrm{~d} m^{2}
$$

For $n=1$ extra dimensions, as in DGP, this gives a propagator of the form $\mathscr{G}(k) \sim 1 / k$ in the IR limit and therefore corresponds to a theory with $\alpha=1 / 2$. For any higher dimensional model, $n \geq 2$, the propagator tends to a constant in the deep IR limit $k \rightarrow 0$ and therefore corresponds to a theory of massive gravity with $\alpha=0$ which exhibits the right behaviour for degravitation which needs $0 \leq \alpha<1 / 2$.

When dealing with more than one extra dimension, great care should however be taken in dealing with divergences that arise on objects of codimension greater or equal to two, see ref. $[4,5]$. The only source on a codimension-two object (such as a cosmic string) that does not give rise to curvature divergences is a pure tension. Any other kind of matter leads to logarithmic divergences on the defect. To avoid such divergences, the defect should first be regularized.

\section{Scalar field toy-model}

To see this, one can study the propagator in a scalar field toy-model. If the scalar field is sourced on a codimension-one,

$$
\partial_{y}^{2} \phi(y)=\delta(y)
$$

the field satisfies $\phi(y) \sim|y|$ and remains regular on the object at $y=0$. If instead the source is localized on a codimension-two surface,

$$
\left(\partial_{y}^{2}+\partial_{z}^{2}\right) \phi(y, z)=\delta^{2}(y, z)
$$

the field configuration is then $\phi(y, z) \sim \log \left(y^{2}+z^{2}\right)$ and hence diverges logarithmically as it approaches the defect $y, z \rightarrow 0$. However if we instead embedded the codimensiontwo object on a codimension-one brane on where a field kinetic term is included,

$$
\left(\partial_{y}^{2}+\partial_{z}^{2}\right) \phi(y, z)=\delta^{2}(y, z)-\delta(z) \partial_{y}^{2} \phi
$$

the field would then be regular on the codimension-two object, [6, 7]. In a sixdimensional setup, this theory follows from the action

$$
S=-\int \mathrm{d}^{6} x \frac{M_{6}^{4}}{2} \partial_{A} \phi \partial^{A} \phi-\int \mathrm{d}^{5} x \frac{M_{5}^{3}}{2} \partial_{\alpha} \phi \partial^{\alpha} \phi-\int \mathrm{d}^{4} x \frac{M_{4}^{2}}{2} \partial_{\mu} \phi \partial^{\mu} \phi,
$$

which corresponds to the effective four-dimensional propagator

$$
\mathscr{G}_{4 d}=\frac{1}{M_{4}^{2}} \frac{1}{k^{2}+m^{2}\left(k^{2}\right)}, \quad \text { with } \quad m^{2}\left(k^{2}\right)=\frac{\pi m_{5}}{4} \frac{\sqrt{m_{6}^{2}-k^{2}}}{\operatorname{arcth}\left(\sqrt{\frac{m_{6}-k}{m_{6}+k}}\right)},
$$


and $m_{6}=M_{6}^{4} / M_{5}^{3}$. From a four-dimensional point of view, this corresponds to a theory of massive gravity with $\alpha=0$. At long wavelengths, $k \ll m_{6}$ the propagator behaves as in six dimensions, while for $m_{6} \ll k \ll m_{5}$, the field effectively sees five dimensions and at shorter wavelengths $k \gg m_{5}$ one recovers a four-dimensional propagator. This behavior has also been obtained in a different codimension-two framework, [8].

This cascading approach that avoids any singularities when dealing with higher codimensions is completely generalizable to any number of dimensions. From a fourdimensional point of view, gravity will then go from four dimensions at small scales, up to five dimensions, etc. up to $(n+4)$ dimensions at large scales, exhibiting a theory of massive gravity with $\alpha=0$.

\section{Cascading Gravity}

The generalization to gravity is straightforward,

$$
S=-\int \mathrm{d}^{6} x \sqrt{-g_{6}} \frac{M_{6}^{4}}{2} R^{(6)}-\int \mathrm{d}^{5} x \sqrt{-g_{5}} \frac{M_{5}^{3}}{2} R^{(5)}-\int \mathrm{d}^{4} x \sqrt{-g_{4}} \frac{M_{4}^{2}}{2} R^{(4)} .
$$

To study the gravitational propagator, one can work around flat space-time, $g_{A B}=\eta_{A B}+$ $h_{A B}$, and decompose the four-dimensional perturbations as $h_{\mu \nu}=h_{\mu \nu}^{T T}+\pi \eta_{\mu \nu}+$ gauge terms. The tensor mode $h_{\mu \nu}^{T T}$ behaves precisely as the scalar field toy-model, and is regularized by the presence of the five-dimensional Einstein-Hilbert term. The scalar mode $\pi$, on the other hand, propagates a ghost. After integrating the sixth dimension, its effective equation on the five-dimensional brane is

$$
-\frac{M_{5}^{3}}{2}\left[\square_{4}-m_{6} \sqrt{-\square_{4}}\right] \pi=\frac{1}{12}\left(T^{(4)}-3 M_{4}^{2} \square_{4} \pi\right)
$$

$\pi$ is therefore finite on the codimension-two brane. However in the UV, $\pi \sim+\square_{4}^{-1} T$ while in the IR, $\pi \sim-T$. The kinetic term hence changes sign signaling the presence of a ghost. This ghost is completely independent to the ghost present in the selfaccelerating branch of DGP but is completely generic to any codimension-two and higher framework with brane localized kinetic terms. In particular this ghost is also present when considering a pure codimension-two scenario with no five-dimensional Einstein-Hilbert term, [9].

There are however two ways to cure the ghost, both of which are natural when considering a realistic higher codimensional scenario. First of all, we do expect a large cosmological constant on the brane, and in the presence of a minimal tension on the brane, the ghost disappears and is replaced by a healthy mode, [6]. Therefore this provides a realistic resolution to the ghost issue and shows that this model of cascading gravity with a brane tension is free of any instability, at least around flat space-time.

Secondly, one never thinks of any defect as being fundamentally thin. Any distributional source, is well described by a delta function at low-energy, but there is nonetheless an energy scale above which the thickness of the brane is resolved, and the brane can no longer be treated as a thin object. This is the case in this cascading scenario, and another 
independent way to cure the ghost is to smooth the brane, [7]. In the underlying theory, each localized Einstein-Hilbert term is nothing but a fundamental six-dimensional scalar curvature object localized on the thick brane. One can check that the ghost disappears and is replaced by a healthy mode when properly taking this into account before taking the thin-brane limit to derive the low-energy effective theory, [7, 9].

When properly taking into account this issue associated with the ghost, we recover a theory of massive gravity composed of one helicity- 2 mode, helicity-one modes that decouple and 2 helicity- 0 modes. In order for this theory to be consistent with standard general relativity in four dimensions, both helicity- 0 modes should decouple from the theory. This decoupling does not happen in a trivial way, and relies on a phenomena of strong coupling. Close enough to any source, both scalar modes are strongly coupled and therefore freeze.

The resulting theory appears as a theory of a massless spin-2 field in four-dimensions, in other words as General Relativity. If $r \ll m_{5}$ and for $m_{6} \leq m_{5}$, the respective Vainshtein scale or strong coupling scale, i.e. the distance from the source $M$ within which each mode is strongly coupled is $r_{i}^{3}=M / m_{i}^{2} M_{4}^{2}$, where $i=5,6$. Around a source $M$, one recovers four-dimensional gravity for $r \ll r_{5}$, five-dimensional gravity for $r_{5} \ll r \ll r_{6}$ and finally six-dimensional gravity at larger distances $r \gg r_{6}$.

\section{Discussion}

Models of massive gravity represent a novel framework for tackling the cosmological constant problem. There is to date only one known ghost-free non-linear realization of massive gravity that does not violate Lorenz invariance, thats is the DGP model (perturbed around its stable branch), and its higher-codimensional generalization: i.e. cascading gravity. Since the DGP model does not exhibit the phenomena of degravitation, cascading gravity represents the only non-linearized explicit candidate for degravitation known so far. Such a theory evades most tests of General Relativity due to strong coupling and is hence compatible with solar systems tests, but represents nonetheless a high-pass filter theory of gravity and slowly filters out in time sources with long wavelength sources such as the cosmological constant.

\section{ACKNOWLEDGMENTS}

This talk is based on work in collaboration with Gia Dvali, Justin Khoury, Stefan Hofmann, Oriol Pujolàs, Michele Redi and Andrew J. Tolley. Research at McMaster is supported by the Natural Sciences and Engineering Research Council of Canada. Research at Perimeter Institute for Theoretical Physics is supported in part by the Government of Canada through NSERC and by the Province of Ontario through MRI.

\section{REFERENCES}

1. G. Dvali, S. Hofmann and J. Khoury, Phys. Rev. D 76, 084006 (2007) [arXiv:hep-th/0703027]. 
2. G. Dvali, New J. Phys. 8, 326 (2006) [arXiv:hep-th/0610013].

3. G. R. Dvali, G. Gabadadze and M. Porrati, Phys. Lett. B 484, 112 (2000) [arXiv:hep-th/0002190]; G. R. Dvali, G. Gabadadze and M. Porrati, Phys. Lett. B 485, 208 (2000) [arXiv:hep-th/0005016].

4. R. Geroch and J. H. Traschen, Phys. Rev. D 36, 1017 (1987).

5. W. D. Goldberger and M. B. Wise, Phys. Rev. D 65, 025011 (2002) [arXiv:hep-th/0104170]; C. de Rham, JHEP 0801, 060 (2008) [arXiv:0707.0884 [hep-th]]; C. de Rham, AIP Conf. Proc. 957, 309 (2007) [arXiv:0710.4598 [hep-th]].

6. C. de Rham, S. Hofmann, J. Khoury and A. J. Tolley, JCAP 0802, 011 (2008) [arXiv:0712.2821 [hep-th]].

7. C. de Rham, G. Dvali, S. Hofmann, J. Khoury, O. Pujolas, M. Redi and A. J. Tolley, Phys. Rev. Lett. 100, 251603 (2008) [arXiv:0711.2072 [hep-th]].

8. N. Kaloper and D. Kiley, JHEP 0705, 045 (2007) [arXiv:hep-th/0703190].

9. G. Gabadadze and M. Shifman, Phys. Rev. D 69, 124032 (2004) [arXiv:hep-th/0312289]. 